\title{
Early Continuous Veno-Venous Hemofiltration Is Effective in Decreasing Intra-Abdominal Pressure and Serum Interleukin-8 Level in Severe Acute Pancreatitis Patients with Abdominal Compartment Syndrome
}

\author{
Jianmin Xu Ying Cui Xiangping Tian \\ Digestive Department, Affiliated Yidu Central Hospital of Weifang Medical College, Qingzhou, China
}

\section{Keywords}

Severe acute pancreatitis - Abdominal compartment syndrome · Continuous veno-venous hemofiltration . Interleukin-8 $\cdot$ Intra-abdominal hypertension

\begin{abstract}
Objective: The aim of this study was to evaluate the efficacy of early continuous veno-venous hemofiltration (CVVH) in decreasing the intra-abdominal pressure (IAP) and serum interleukin-8 (IL-8) level in severe acute pancreatitis (SAP) patients with abdominal compartment syndrome (ACS). Methods: Twenty-five ACS patients of SAP were enrolled in a prospective study conducted according to the standard management protocol. They were treated in the intensive care unit (ICU) of Affiliated Yidu Central Hospital of Weifang Medical College and underwent CVVH. Eleven patients were set up as the control group that received no hemofiltration and surgical treatment due to economic or other reasons but solely conventional treatment. Serum amylase, liver and kidney function, and $C$ reactive protein were investigated before and after treatment. IAP and blood level of IL-8 were measured daily to investigate their time course of changes and the correlation between the 2 parameters. Results: Se-
\end{abstract}

\section{KARGER}

(C) 2017 S. Karger AG, Basel

E-Mail karger@karger.com

www.karger.com/bpu rum amylase levels, C-reactive protein and IAP were significantly lower and liver and kidney function was significantly better than those of the control group $(p<0.05)$. IAP on admission to the ICU was high, at $22.9 \pm 2.1 \mathrm{~mm} \mathrm{Hg}$. The IAP was significantly lower to $17.2 \pm 2.2 \mathrm{~mm} \mathrm{Hg}(p<0.01) 24 \mathrm{~h}$ after the initiation of $\mathrm{CVVH}$, and thereafter decreased rapidly. The average blood level of IL- 8 was high at $88.2 \pm 25.1 \mathrm{ng} / \mathrm{L}$ on admission. However, it significantly decreased to $63.2 \pm 18.7$ $\mathrm{ng} / \mathrm{L}(p<0.01) 24 \mathrm{~h}$ after the initiation of CVVH, and subsequently decreased. There was a significant positive correlation between the blood level of IL-8 and IAP $(r=0.62, p<0.01)$. Conclusions: $\mathrm{CVVH}$ is effective to decrease the IAP and the blood level of IL-8 in ACS patients of SAP. The blood level of IL-8 was significantly correlated with IAP, suggesting that IL-8 might play an important role in the pathogenesis of ACS. Early CVVH appeared to be effective in the treatment of ACS in patients with SAP through the removal of causative cytokines such as IL-8, and it thereby decreased interstitial edema to lower IAP and should be applied in the early stage of ACS. Video Journal Club 'Cappuccino with Claudio Ronco' at http://www.karger.com/?doi=480223. @ 2017 S. Karger AG, Basel

J.X. and Y.C. contributed equally to this work.
Jianmin Xu

Digestive Department, Affiliated Yidu Central Hospital of Weifang Medical College No. 4138 Linglongshan South Road

Qingzhou City, Shandong Province (China)

E-Mail jianminxu1977@163.com 


\section{Introduction}

Severe acute pancreatitis (SAP) is most commonly characterized by cytokine activation, pancreatic necrosis, systemic inflammatory response syndrome (SIRS), and multiple organ dysfunction syndrome (MODS) $[1,2]$. It is considered to be a manifestation of organ dysfunction resulting from the exacerbation of SIRS triggered by local inflammation in the pancreas. The fundamental pathophysiology of SIRS is hypercytokinemia, a pathological condition in which inflammatory cytokines are excessively released from immunocompetent cells and their blood levels increase. Interleukin-8 (IL-8) is one of inflammatory cytokines triggering SIRS and may activate various humoral mediator cascades that cause organ dysfunction [3].

Abdominal compartment syndrome (ACS), a lethal complication of SAP, is defined as the combination of intra-abdominal pressure (IAP) $>20 \mathrm{~mm} \mathrm{Hg}$ and new-onset organ failure (OF) or acute worsening of existing OF [3]. The pathophysiology of ACS is considered to be directly associated with the pancreas inflammation, which initiates a cascade of acute peripancreatic fluid collections (APFC), capillary leakage syndrome (CLS), and paralytic ileus leading to an elevated IAP $[4,5]$. Several studies describing the effect of various interventions to lower IAP in SAP patients with both medical and surgical measures have been published in recent years $[6,7]$. Despite all these evidence-based advances in the evaluation and treatment of SAP patients with ACS, ACS remains a life-threatening complication. In previous studies $[7,8]$, continuous veno-venous hemofiltration $(\mathrm{CVVH})$ was found to efficiently remove various humoral mediators to prevent the deterioration of SIRS to the MODS in SAP, and have reported its efficacy in SAP patients $[9,10]$. But, there are a few reports regarding ACS therapy with CVVH.

So, we conducted a retrospective study to assess the efficacy of CVVH in the treatment of ACS in SAP patients and probed the relationship between IL- 8 and IAP in these patients to prospectively evaluate whether cytokine (such as IL-8) removal with CVVH is effective in treating of ACS in SAP.

\section{Materials and Methods}

\section{Patients and Sample Collection}

Twenty-five patients with SAP who were admitted to the intensive care unit (ICU) of Affiliated Yidu Central Hospital of Weifang Medical College within $72 \mathrm{~h}$ after the onset of the disease were collected as the treatment group. Other 11 patients were set up as the control group that received no hemofiltration and surgical treatment due to economic or other reasons but solely conventional treatment. Serum amylase, liver and kidney function, and C-reactive protein were investigated before and after treatment in both groups. The study period extended from January 2013 to December 2015. Inclusion criteria for SAP were defined according to the Atlanta criteria [11]. The study protocol was in agreement with the guidelines of the Ethics Committee at our hospital. The severity of SAP was evaluated according to the Ranson score, acute physiology and chronic health evaluation score II, CTgrade. The blood level of IL-8 was determined daily in a clinical laboratory using a rapid measurement. IAP exceeded more than $20 \mathrm{~mm} \mathrm{Hg}$ on admission in all cases, demonstrating that they already had ACS.

\section{Continuous Veno-Venous Hemofiltration}

Continuous veno-venous hemodiafiltration was performed using Diapact CRRT B. Braun Co. (Melsungen, Germany). Synthetic high-volume membrane filters with a surface area of 1.5-2.2 $\mathrm{m}^{2}$ were used and changed every $24 \mathrm{~h}$ or when filter blocking occurred. Vascular access was obtained by a double- or triple-lumen catheter using the femoral or jugular vein. Anticoagulation was provided with non-fractioned heparin, adjusting the dosage according to the value of activated partial thromboplastin time in the plasma. The procedure was performed with a low dose of heparin or without it when possible. The substitution fluid infusion rate was $1,250-1,750 \mathrm{~mL} / \mathrm{h}$ in a pre-diluted or post-diluted manner, comprising 30-42 L of the total substitute in $24 \mathrm{~h}$. The blood flow rate was $50-200 \mathrm{~mL} / \mathrm{min}$. The ultrafiltration rate was adjusted according to the diuresis and fluid balance.

\section{Other Treatments}

All patients were treated with fasting, gastrointestinal decompression, acid suppression therapy. Synthetic protease inhibitor, gabexate mesilate (300 mg/day; FOY, Changzhou Pharmaceutical Co., Jiangsu, China) was administered with systemic continuous infusion and octreotide $(0.3 \mathrm{mg}$, Novartis Pharmaceutical Co., Switzerland) were dissolved into the saline $(48 \mathrm{~mL})$ continually infused twice a day for the treatment of pancreatitis. Fluid, electrolytes, albumin, and insulin were replaced depending on central venous pressure $\left(6-10 \mathrm{~mm} \mathrm{H}_{2} \mathrm{O}\right)$, hematocrit (30$35 \%)$, urinary excretion, and blood glucose measurement. All patients received systemic administration of antibiotics to prevent infectious complications. Nutritional support was initially started with total parenteral nutrition, which was later combined with enteral feeding as soon as gastrointestinal peristaltic movement was confirmed. Patients who developed acute respiratory distress syndrome underwent tracheal intubation to enable ventilatory support according to the protective-ventilation strategy [12].

Measurements of IAP and Determination of Blood Level of IL-8 IAP was measured with a catheter inserted into the bladder, according to the standard technique established by WSACS in 2006 [13]. Twenty-five milliliter of $0.9 \% \mathrm{NaCl}$ was instilled, and the midaxillary line was considered level 0 . The IAP was determined every $12 \mathrm{~h}$ in the intensive care. All patients underwent consecutive IAP monitoring for at least 7 days. Serum levels of IL- 8 were determined by enzyme-linked immunosorbent assay (Rapidbio 
Table 1. Changes of patients' serum biochemical indexes in 2 groups before and after treatment of 7 days

\begin{tabular}{|c|c|c|c|c|c|c|}
\hline & \multicolumn{2}{|c|}{ Amylase, $10^{3} \mathrm{U} / \mathrm{L}$} & \multicolumn{2}{|l|}{$\mathrm{ALT}, \mathrm{U} / \mathrm{L}$} & \multicolumn{2}{|l|}{$\mathrm{Cr}, \mu \mathrm{mol} / \mathrm{L}$} \\
\hline & before & after & before & after & before & after \\
\hline Treatment group & $1.82 \pm 0.73$ & $0.19 \pm 0.21^{*, \#}$ & $225.7 \pm 19.3$ & $55.6 \pm 23.2^{*, \#}$ & $288.9 \pm 10.7$ & $102.0 \pm 37.4^{*, \#}$ \\
\hline \multirow[t]{3}{*}{ Control group } & $1.89 \pm 0.59$ & $0.64 \pm 0.27^{*}$ & $218.7 \pm 19.2$ & $133.4 \pm 25.6^{*}$ & $291.1 \pm 10.9$ & $229.7 \pm 35.7^{*}$ \\
\hline & \multicolumn{2}{|l|}{$\mathrm{CRP}, \mathrm{mg} / \mathrm{L}$} & \multicolumn{2}{|l|}{$\mathrm{Hb}, \mathrm{g} / \mathrm{L}$} & \multicolumn{2}{|l|}{ ALB } \\
\hline & before & after & before & after & before & after \\
\hline Treatment group group & $54.6 \pm 6.4$ & $22.3 \pm 2.5^{*, \#}$ & $116.3 \pm 11.6$ & $114.6 \pm 11.2$ & $35.8 \pm 6.73$ & $33.25 \pm 6.23^{*, \#}$ \\
\hline \multirow[t]{3}{*}{ Control group } & $56.9 \pm 6.5$ & $45.4 \pm 4.2^{*}$ & $115.9 \pm 10.8$ & $114.3 \pm 11.8$ & $34.9 \pm 6.65$ & $30.10 \pm 5.84^{*}$ \\
\hline & \multicolumn{2}{|l|}{$\mathrm{Ca}$} & \multicolumn{2}{|l|}{ WBC } & \multicolumn{2}{|l|}{ INR } \\
\hline & before & after & before & after & before & after \\
\hline Treatment group & $1.94 \pm 2.12$ & $2.11 \pm 1.85^{*}$ & $15.6 \pm 4.32$ & $10.3 \pm 3.21^{*, \#}$ & $1.14 \pm 0.16$ & $1.17 \pm 0.18$ \\
\hline Control group & $1.96 \pm 1.99$ & $2.08 \pm 1.76^{*}$ & $15.1 \pm 4.61$ & $11.9 \pm 2.78^{*}$ & $1.15 \pm 0.18$ & $1.18 \pm 0.15$ \\
\hline
\end{tabular}

${ }^{*} p<0.05$ vs. before treatment; ${ }^{\#} p<0.05$ vs. control group.

Company, USA) and expressed in ng/L. IL-8 was measured first on admission to the ICU, and thereafter daily at 6 am in principle until discharge from the ICU.

\section{Statistics}

Values are expressed with means \pm SD. Daily changes in mean blood levels of IL- 8 and IAP were tested by repeated measure oneway analysis of variance with a post hoc test using Fisher's protected least significant difference. The correlation between 2 parameters was tested by simple regression analysis. A $p$ value $<0.05$ was considered significant. SPSS 13.0 software application was used for all analysis.

\section{Results}

Changes of patients' serum biochemical indexes in 2 groups before and after treatment of 7 days are shown in Table 1. The biochemical parameters such as serum amylase, calcium, creatinine, and CRP in the 2 groups were significantly ameliorated compared with their values before treatment, but the improvement was more obvious in the treatment group. Of the 25 patients who had received intensive care including $\mathrm{CVVH}$ in the present study, 23 patients recovered and were eventually discharged. Two patients gradually deteriorated and therefore underwent open abdomen management. One died of multiple OF and the other died from septicopyemia. There were 4 patients who died of multiple OF in the control group.
IAP began to decline on day 3 in the control group, but in the treatment group, IAP had decreased significantly since day 1 upon CVVH treatment. Also, IAP dropped from day 1 continuously to day 6 in the treatment group, which was statistically significant in comparison to the results before treatment. By day 6 , IAP of the treatment group had fallen into about the normal range. IAP values recorded on days $1,2,3,4,5$, and 6 after CVVH treatment were significantly lower than those of the control group $(p<0.05)$. The time course of the changes in IAP during the 7 days following the initiation of treatment in 2 groups is shown in Figure 1.

Serum IL-8 levels were significantly increased in the 2 groups before treatment. After treatment, the serum IL- 8 levels decreased gradually in the 2 groups, but decreased more significantly in the treatment group. Differences of IL- 8 levels between the 2 groups at the same time point were statistically significant $(p<0.05)$. The time course of the change in blood level of IL- 8 in 2 groups during the 7 days of initiation of treatment is shown in Figure 2.

The correlation between blood level of IL- 8 and simultaneously measured IAP is shown in Figure 3. The blood level of IL- 8 was found to be significantly and positively correlated with IAP $(r=0.62, p<0.01)$. Figure 4 shows the correlation between changes in IL- 8 ( $\Delta$ IL- 8 ; difference between the value of IL- 8 before treatment and corresponding value after 3 days of treatment with CVVH) and changes in IAP ( $\triangle \mathrm{IAP}$; difference between IAP before 


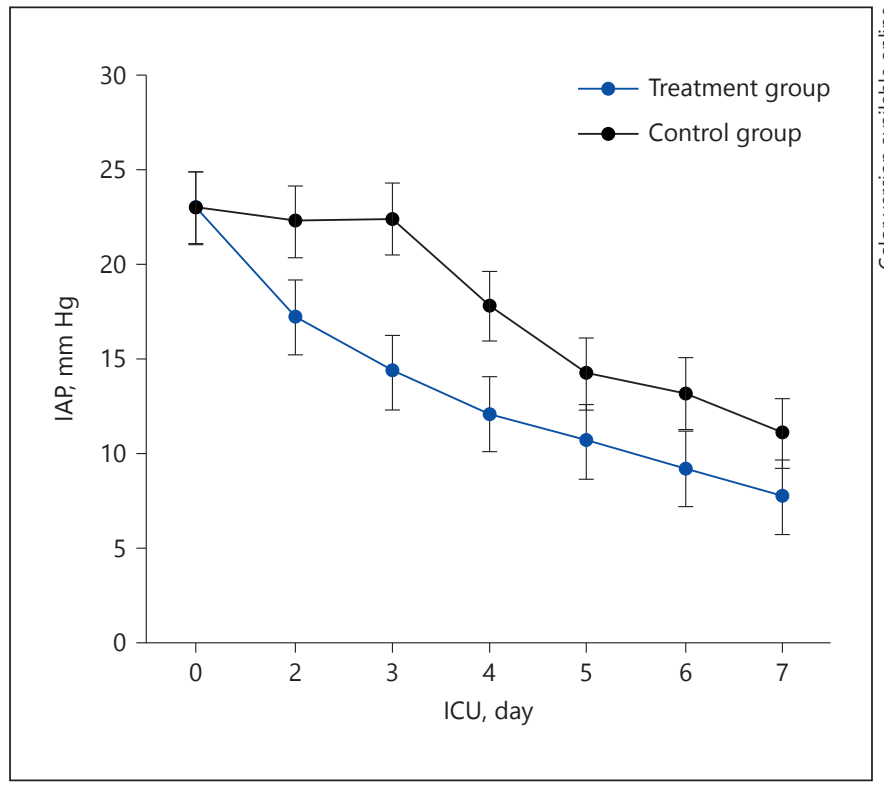

Fig. 1. Time course of changes in IAP in patients with severe acute pancreatitis in 2 groups during 7 days after admission.

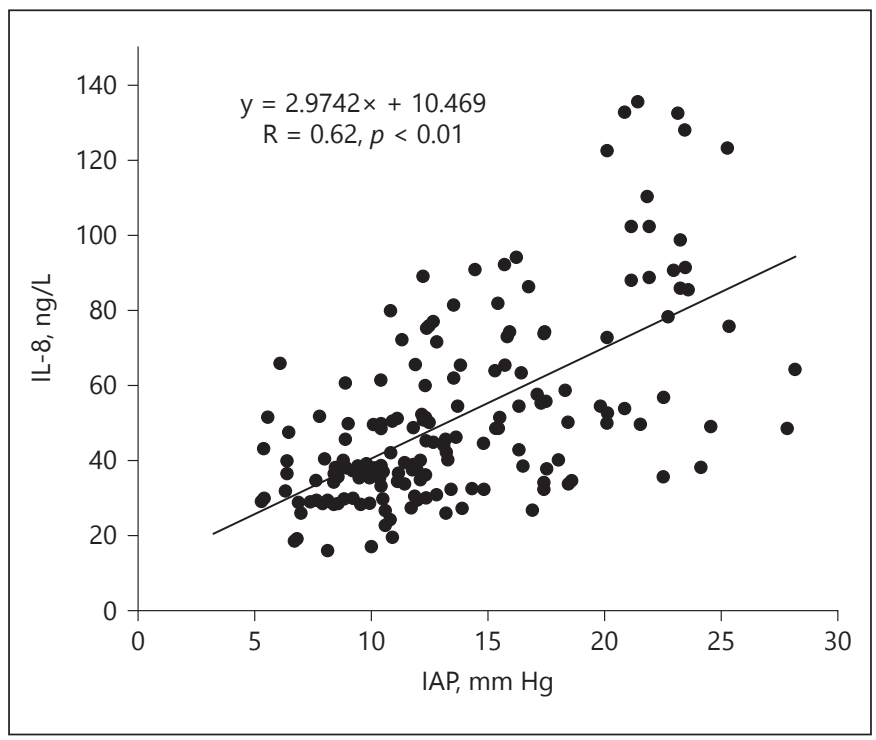

Fig. 3. The correlation between IL- 8 and simultaneously measured IAP.

treatment and 3 days after treatment). There was a significant correlation between the $2(r=0.67, p<0.01)$.

Figure 5 shows the correlation between cumulative water balance (CWB) during the 3 days after treatment and $\triangle \mathrm{IAP}$. There was no correlation between CWB and $\triangle \mathrm{IAP}$.

CVVH Treat ACS of SAP

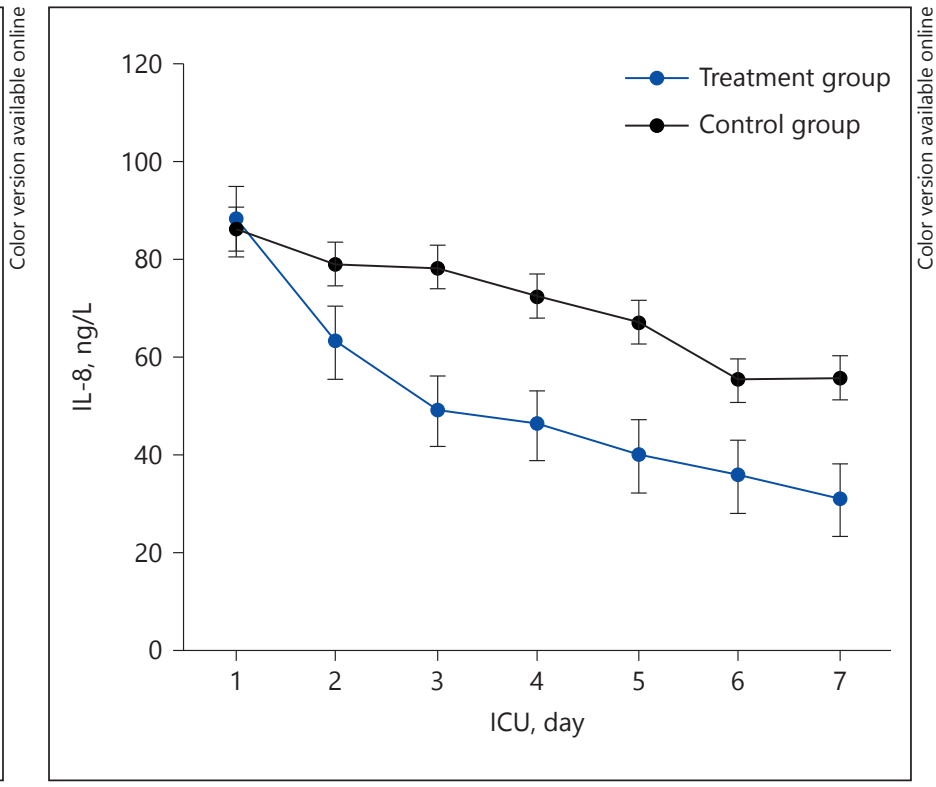

Fig. 2. Time course of changes in blood levels of IL-8 in patients with severe acute pancreatitis in 2 groups during 7 days after admission.

\section{Discussion}

SAP is characterized by the development of local pancreatic complications, OF, or both. It has been widely accepted that SAP is a typical SIRS caused by local inflammation in the pancreas $[14,15]$. SAP injures the pancreas itself as well as the surrounding organs. In SAP, several inflammatory mediators contribute to increased capillary permeability in various organs, which together with aggressive fluid resuscitation may result in visceral edema and the development of intra-abdominal hypertension and at last develop ACS $[16,17]$. ACS, which is defined as a sustained IAP greater than $20 \mathrm{~mm}$ $\mathrm{Hg}$ that is associated with the development of organ dysfunction or failure [18], is the most severe form of acute pancreatitis. Studies show that $11-30 \%$ of SAP patients have ACS, which has a high mortality rate of 30 $60 \%$, despite significant improvement in treatment due to the better understanding of the pathophysiology of disease, early aggressive fluid resuscitation, and timely surgical intervention. At present, it is well accepted that SAP patients with SIRS have a high risk of developing ACS [19]. Blood levels of various cytokines are elevated in SAP and among them, the blood level of IL-8 as one of main inflammatory cytokines has been reported to reflect the severity of SAP. But, there are a few reports regarding the correlation between the blood level of 


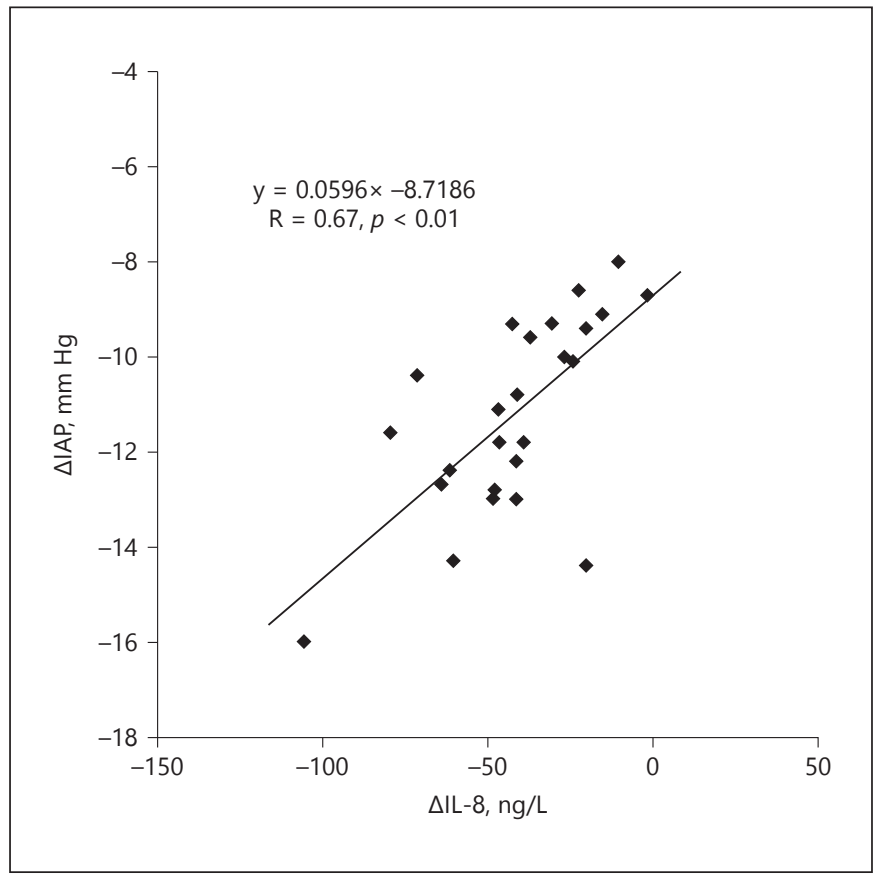

Fig. 4. The correlation between $\Delta \mathrm{IL}-8$ (difference between the value of IL- 8 before treatment and corresponding value after 3 days of treatment with $\mathrm{CVVH}$ ) and $\triangle \mathrm{IAP}$ (difference between the value of intra-abdominal pressure before treatment and 3 days after treatment).

IL-8 and IAP. In many studies, CVVH was found to efficiently remove various humoral mediators such as IL-8 in the blood stream to prevent the deterioration of SIRS and that thereby it is useful for preventing or treating MODS $[20,21]$. However, the clinical efficacy of CVVH in the treatment of SAP with ACS and its mechanism in reducing IAP have not been reported in the literature. So we have performed CVVH to treat ACS in SAP patients in combination with systemic administration of gabexate mesilate and octreotide for the treatment of pancreatitis, and in combination with the administration of antibiotics for the prevention of infectious complications.

In this study, patients with SAP had developed ACS on admission. The blood level of IL- 8 was also high, but it was rapidly lowered after the initiation of CVVH. During the treatment of $\mathrm{CVVH}$, fluid balance was strictly controlled with continuous water removal through filtration by CVVH to maintain circulating blood volume and avoid multiple OF. Is the decrease of IAP is associated with limiting water intake? Our research found that there was no correlation between CWB and change in IAP, so the water removal has not contributed to the low-

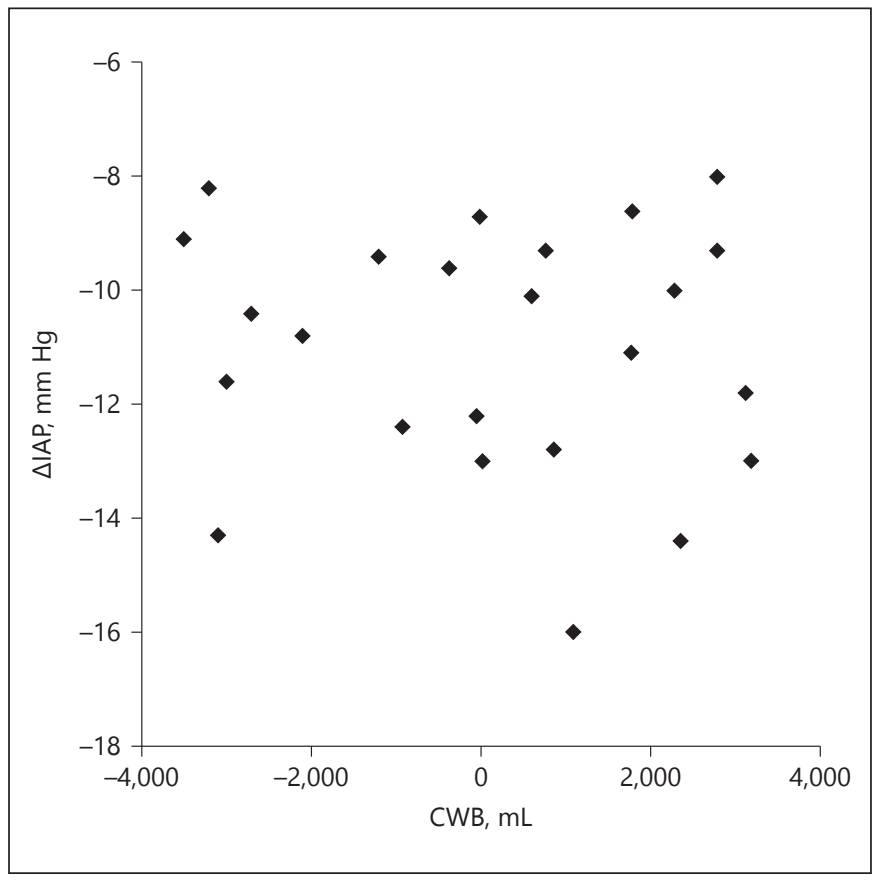

Fig. 5. The correlation between cumulative water balance (CWB) during 3 days after initiation of treatment and $\triangle \mathrm{IAP}$ (difference between the value of intra-abdominal pressure before treatment and 3 days after treatment).

ering of IAP, which is consistent with the findings of the research conducted by Oda et al. [9]. Other treatment methods affecting IAP, such as abdominal drainage to remove peritoneal fluid, has not been applied. So IAP was significantly decreased mainly due to CVVH. Correlation analysis showed there was a significant positive correlation between the blood level of IL- 8 and IAP. And changes before and after treatment in IL- 8 levels were also significantly correlated with changes in IAP. Furthermore, there was no correlation between CWB and changes in IAP, so we are of the opinion that elevated IL-8 is one of the inflammatory cytokines contributing to ACS. Removal of causative humoral mediators such as IL-8 due to CVVH application improved vascular permeability and subsequent fluid refilling from the interstitium-lowered IAP. Twenty-three of 25 patients were successfully recovered from ACS without exacerbation and were discharged from the hospital, yielding a survival rate of $92 \%$. But in the control group, 4 of 11 patients died due to multiple OF. These results suggest that CVVH, which can effectively remove cytokines and thereby regulate SIRS, is effective in the treatment of ACS in patients with SAP. 
In our study, 2 patients in the treatment group gradually deteriorated and therefore underwent open abdomen management. One died of multiple OF and the other died from septicopyemia. At present, it has been widely accepted that most cases of ACS with SAP should be treated with non-surgical supportive care, and that surgery should be performed only when patients' symptoms are complicated with infected pancreatic necrosis. Furthermore, there is no uniform consensus on the indications for surgical decompression in ACS associated with SAP. Our study suggested that most cases of ACS with SAP recovered well through early CVVH, so we suggest that ACS of SAP should be applied to CVVH even in the early stage.

\section{Conclusions}

Our study confirmed that CVVH significantly decreased the IAP and the blood level of IL-8 in ACS patients of SAP. The blood level of IL- 8 was significantly correlated with IAP, suggesting that IL-8 may play an impor- tant role in the pathogenesis of ACS. CVVH effectively lowered the blood level of IL-8 and significantly reduced IAP by removing cytokines, thereby controlling SIRS. So we think that CVVH is effective for preventing and treating ACS patients of SAP, and that it should be applied in the early stage of ACS. Furthermore, the need for surgical interventions in ACS patients can be decreased with the improvement of nonsurgical intervention techniques. The underlying mechanism of ACS demands for further clarification in a prospective multicenter study.

\section{Acknowledgments}

We thank the central laboratory for providing technical support and we are grateful to the ICU of the Affiliated Yidu Central Hospital of Weifang Medical College for their valuable cooperation.

\section{Disclosure Statement}

The authors have no conflicts of interest to declare.

\section{References}

1 Malbrain ML, Cheatham ML, Kirkpatrick A, Sugrue M, Parr M, De Waele J, Balogh Z, Leppäniemi A, Olvera C, Ivatury R, D’Amours S, Wendon J, Hillman K, Johansson K, Kolkman $\mathrm{K}$, Wilmer A: Results from the international conference of experts on intra-abdominal hypertension and abdominal compartment syndrome. I. Definitions. Intensive Care Med 2006;32:1722-1732.

2 Mole DJ, Gungabissoon U, Johnston P, Cochrane L, Hopkins L, Wyper GM, Skouras C, Dibben C, Sullivan F, Morris A, Ward HJ, Lawton AM, Donnan PT: Identifying risk factors for progression to critical care admission and death among individuals with acute pancreatitis: a record linkage analysis of Scottish healthcare databases. BMJ Open 2016;6: e011474.

3 Sendler M, Dummer A, Weiss FU, Krüger B, Wartmann T, Scharffetter-Kochanek K, van Rooijen N, Malla SR, Aghdassi A, Halangk W, Lerch MM, Mayerle J: Tumour necrosis factor a secretion induces protease activation and acinar cell necrosis in acute experimental pancreatitis in mice. Gut 2013;62: 430-439.

4 Leppaniemi A, Mentula P, Hienonen P, Kemppainen E: Transverse laparostomy is feasible and effective in the treatment of abdominal compartment syndrome in severe acute pancreatitis. World J Emerg Surg 2008; 3:6.
5 Jaipuria J, Bhandari V, Chawla AS, Singh M: Intra-abdominal pressure: time ripe to revise management guidelines of acute pancreatitis? World J Gastrointest Pathophysiol 2016;7: 186-198.

6 Mentula P, Hienonen P, Kemppainen E, Puolakkainen P, Leppäniemi A: Surgical decompression for abdominal compartment syndrome in severe acute pancreatitis. Arch Surg 2010;145:764-769.

7 Okita Y, Okahisa T, Sogabe M, Suzuki M, Ohnishi Y, Ito S: Low-volume continuous hemodiafiltration with nafamostat mesilate increases trypsin clearance without decreasing plasma trypsin concentration in severe acute pancreatitis. ASAIO J 2007;53:207212.

8 Wang HL, Yu KJ: Sequential blood purification therapy for critical patients with hyperlipidemic severe acutepancreatitis. World J Gastroenterol 2015;21:6304-6309.

9 Oda S, Hirasawa H, Shiga H, Matsuda K, Nakamura M, Watanabe E, Moriguchi T: Management of intra-abdominal hypertension in patients with severe acute pancreatitis with continuous hemodiafiltration using a polymethyl methacrylate membrane hemofilter. Ther Apher Dial 2005;9:355-361.

10 Wang S, Xu L, Feng X, Li S, Feng Q, Liu C, Zhang $\mathrm{X}$, Zhao Q: Is continuous venovenous hemofiltration effective against severe acute pancreatitis? Artif Organs 2013;37:615-622.
11 Bradley EL 3rd: A clinically based classification system for acute pancreatitis. Summary of the international symposium on acute pancreatitis, Atlanta, GA, September 11-13, 1992. Arch Surg 1993;128:586-590.

12 Amato MB, Barbas CS, Medeiros DM, Magaldi RB, Schettino GP, Lorenzi-Filho G, et al: Effect of a protective-ventilation strategy on mortality in the acute respiratory distress syndrome. N Engl J Med 1998;338:347354.

13 Malbrain ML, Cheatham ML, Kirkpatrick A, Sugrue M, Parr M, De Waele J, Balogh Z, Leppäniemi A, Olvera C, Ivatury $R$, D’Amours S, Wendon J, Hillman K, Johansson K, Kolkman K, Wilmer A: Results from the international conference of experts on intra-abdominal hypertension and abdominal compartment syndrome. I. Definitions. Intensive Care Med 2006;32:17221732.

14 Gunjaca I, Zunic J, Gunjaca M, Kovac Z: Circulating cytokine levels in acute pancreatitismodel of SIRS/CARS can help in the clinical assessment of disease severity. Inflammation 2012;35:758-763.

15 Malmstrom ML, Hansen MB, Andersen AM, Ersboll AK, Nielsen OH, Jorgensen LN, Novovic S: Cytokines and organ failure in acute pancreatitis: inflammatory response in acute pancreatitis. Pancreas 2012;41:271277. 
16 Trikudanathan G, Vege SS: Current concepts of the role of abdominal compartment syndrome in acute pancreatitis - an opportunity or merely an epiphenomenon. Pancreatology 2014;14:238-243.

17 De Waele JJ, De Laet I, Kirkpatrick AW, Hoste E: Intra-abdominal hypertension and abdominal compartment syndrome. Am J Kidney Dis 2011;57:159-169.

18 Radenkovic DV, Johnson CD, Milic N, Gregoric $\mathrm{P}$, Ivancevic $\mathrm{N}$, Bezmarevic $\mathrm{M}$, Bilanovic
D, Cijan V, Antic A, Bajec D: Interventional treatment of abdominal compartment syndrome during severe acute pancreatitis: current status and historical perspective. Gastroenterol Res Pract 2016;2016:5251806.

19 Chu LP, Zhou JJ, Yu YF, Huang Y, Dong WX: Clinical effects of pulse high-volume hemofiltration on severe acute pancreatitis complicated with multiple organ dysfunction syndrome. Ther Apher Dial 2013;17: 78-83.
20 Ke L, Ni HB, Tong ZH, Li WQ, Li N, Li JS: Intra-abdominal pressure and abdominal perfusion pressure: which is a better marker of severity in patients with severe acute pancreatitis. J Gastrointest Surg 2011;15:14261432.

21 Sun IF, Lee SS, Lin SD, Lai CS: Continuous arteriovenous hemodialysis and continuous venovenous hemofiltration in burn patients with acute renal failure. Kaohsiung J Med Sci 2007;23:344-351. 